\title{
DESAFIOS E DISPARIDADES DA INICIAÇÃO CIENTÍFICA NOS CURSOS DE ENGENHARIA DE MINAS E ENGENHARIA METALÚRGICA DA UEMG/ UNIDADE JOÃO MONLEVADE
}

\author{
J. S. Alexandrino ${ }^{1}$, T. E. D. Ferreira ${ }^{2}$, K. C. Ferreira ${ }^{3}$, G.A. Torres $^{4}$, F. M. Gonçalves ${ }^{5}$ \\ Universidade do Estado de Minas Gerais/UEMG - Unidade João Monlevade \\ juniaalexandrino@yahoo.com.br ${ }^{1}$
}

Submetido 10/11/2017 - Aceito 17/11/2017

DOI: $10.15628 /$ holos.2017.6383

\section{RESUMO}

A formação do engenheiro no Brasil está crescendo de forma significativa e, com isso, a necessidade de realizar pesquisas se torna cada vez mais importante para os estudantes. O mercado da mineração e metalurgia passa por altos e baixos, por isso é sempre enriquecedor trabalhar com projetos, seja ele de pesquisa ou de extensão, pois é uma forma de aprender a trabalhar, de estudar e de crescer profissionalmente. Atentando-se às disparidades apresentadas pelos dois cursos estudados, no que diz respeito à participação em projetos de pesquisa, os resultados mostraram o maior engajamento de discentes dos cursos de Engenharia de Minas em detrimento dos de Engenharia Metalúrgica. Entretanto, essa parcela ainda é baixa considerando-se o número total de alunos dos cursos estudados. Adicionalmente, a melhor divulgação e o maior envolvimento dos professores nos projetos, foram identificados como os principais desafios recorrentes ao acesso à iniciação científica pelos alunos da referida instituição ensino.

PALAVRAS-CHAVE: ensino, engenharia, pesquisa, crescimento.

\section{CHALLENGES AND DISPARITIES OF SCIENTIFIC INITIATION IN THE MINING AND METALLURGICAL ENGINEERING PROGRAMS OF UEMG / JOÃO MONLEVADE CAMPUS}

\begin{abstract}
The formation of the engineer in Brazil is growing significantly and, with this, the need in conducting research becomes increasingly important for the students. The mining and metallurgical market goes through ups and downs, so it is always enriching to work with projects, both research and extension, as it is a way to learn how to work, to study and to grow professionally. Considering the disparities presented by the two courses studied, regarding the participation in
\end{abstract}

research projects, the results showed a greater engagement of Mining Engineering students in detriment of those of Metallurgical Engineering. However, this portion is still low considering the total number of students in the courses studied. In addition, a better dissemination and greater involvement of teachers in the projects were identified as the main recurring challenges to access scientific initiation by the students of the referred institution

KEYWORDS: education, engineering, research, growing. 


\section{INTRODUÇÃO}

O desenvolvimento da história do ensino de Engenharia, no Brasil, quando comparado ao de outros países do mundo, permaneceu em atraso por aproximadamente um século. Isso aconteceu em razão da origem escravocrata da economia nacional, onde se acreditava que uma mão-de-obra barata implicava na proibição da instalação de indústrias (Bazzo, 1998).

As dimensões técnica, generalista, humanística e crítica esperada na formação do engenheiro devem permitir, conforme a Resolução do Conselho Nacional de Educação - CNE - de $11 / 03$ / 2002, a construção de um novo perfil do profissional de engenharia, que considere não somente a capacidade de propor soluções tecnicamente corretas, mas também a ambição de considerar os problemas em sua totalidade, a base filosófica com enfoque na competência, a preocupação com a valorização do ser humano e a preservação do meio ambiente, e a integração social e política (Tonini, 2007).

A atual recessão econômica do mercado brasileiro impacta diretamente sobre o mercado profissional, especialmente na área da engenharia. Contudo, os profissionais mais afetados são os engenheiros recém-formados, que possuem pouca experiência ou muitas vezes são pouco qualificados. Visto as dificuldades e o gasto para se investir em qualificação de mão-de-obra em curto prazo, um dos principais problemas que se tem observado é a falta de cursos adequados. Portanto, tal falta de experiência pode ser evitada com a possibilidade de trabalhar com projetos de pesquisa, extensão e estágios.

\section{REVISÃO BIBLIOGRÁFICA}

Apesar das instituições de ensino não serem obrigadas a oferecer pesquisa em suas estruturas curriculares, estas são cientes que devem difundir o conhecimento que produzem, fundamentados em ampla pesquisa e extensão, levando à evolução do próprio conhecimento, como também à melhoria dos serviços oferecidos. Por conseguinte, o aumento na participação em projetos de pesquisa, por parte dos alunos, pode ser um aliado no aprimoramento das suas habilidades, gerando também novas qualificações (Gomes et al, 2004).

A iniciação científica é classificada como um instrumento de formação que possibilita introduzir a pesquisa científica aos estudantes de graduação, permitindo ao aluno um apoio teórico e metodológico que contribua na sua formação profissional e pessoal. Tem a finalidade de despertar vocação científica, estimulando potenciais talentos entre os estudantes de graduação, envolvidos em projetos de pesquisa, orientados por pesquisador qualificado (Bastos et al., 2010). É por esse motivo que os docentes devem incentivar a pesquisa, para que o discente desenvolva a postura crítica, de modo geral, apresentando mais conhecimento em áreas específicas.

A iniciação científica pode ser também compreendida, em uma perspectiva mais ampla, como um processo que abarca "todas as experiências vivenciadas pelo aluno, numa instituição educacional, com o objetivo de desenvolver a chamada formação cientifica" e "todas as 
oportunidades de participação ou de envolvimento do aluno com o pesquisar durante a graduação" (Simão et al. p. 91, 1996).

As atividades de pesquisa científica são de suma importância na estruturação e consolidação do conhecimento para a formação do engenheiro. Para Tonini (2007), a indissociabilidade entre a tríade ensino, pesquisa e extensão é primordial para o processo de conhecimento do aluno, pois possibilita e fortalece a relação entre teoria e prática, discentes e docentes, compromissos sociais, ambientais e éticos e o desenvolvimento de novas tecnologias. Neste contexto, a iniciação científica transformou-se em uma atividade estratégica para o país, pois a possibilidade de muitos dos atuais iniciantes em ciência se tornarem pesquisadores no futuro, propicia benefícios na área da ciência para o Brasil.

A Universidade do Estado de Minas Gerais - Unidade João Monlevade iniciou suas atividades em setembro de 2006. Os primeiros cursos a serem oferecidos pela Instituição foram Engenharia Ambiental e Engenharia de Minas. Posteriormente, vieram os cursos de Engenharia Metalúrgica e de Engenharia Civil. A criação e ampliação do ensino aumentou a oferta de ensino superior gratuito e de qualidade na cidade mineira de João Monlevade, localizada a 110 quilômetros de Belo Horizonte.

Portanto, este trabalho propõe identificar e mensurar o nível de engajamento dos discentes em iniciação científica nos cursos de graduação de Minas e Metalurgia da UEMG.

\section{METODOLOGIA}

A pesquisa ficou restrita aos cursos de graduação em Engenharia de Minas e de Engenharia Metalúrgica, da UEMG Unidade de João Monlevade, pois os cursos estudados têm significativa participação no número de engenheiros formados anualmente na região.

Primeiramente, foi realizado um levantamento do número de graduados e graduandos que participaram em atividades de iniciação científica, desde o ano de 2012 até o primeiro semestre de 2016, nos cursos citados. A execução desse levantamento contou com o apoio da coordenadoria do Centro de Pesquisa e Extensão da UEMG. Além disso, a Plataforma Lattes também foi utilizada para fins de investigação, visto que esta oferece subsídios para identificar indiretamente o envolvimento dos alunos nas ações de iniciação científica.

Tendo em vista o desenvolvimento dos projetos de pesquisa realizados pelos alunos de engenharia da UEMG, para fins de verificação e participação, os seguintes dados também foram investigados através da Plataforma Lattes:

a) Número de alunos que desenvolveram e desenvolvem projetos de Iniciação Científica;

b) Publicações em periódicos;

c) Publicações completas em congresso nacionais e internacionais;

d) Apresentações em congresso nacionais e internacionais;

e) Publicações de resumos;

f) Publicação de capítulos de livros. 
Adicionalmente, um questionário foi aplicado aos alunos do décimo período de cada um dos dois cursos de graduação estudados nesta pesquisa, com o objetivo de indicar o grau de participação desses discentes em projetos de pesquisa, visto que estiveram matriculados durante todo o período em que esta pesquisa se comprometeu a estudar. Após essa pesquisa, foram avaliados os motivos das disparidades entre os dois cursos.

\section{RESULTADOS E DISCUSSÕES}

O gráfico representado na Figura 1 apresenta o número de estudantes em projetos de iniciação cientifica no período de 2012 ao primeiro semestre de 2016, para cada um dos dois cursos de graduação estudados da Universidade do Estado de Minas Gerais - Unidade João Monlevade.

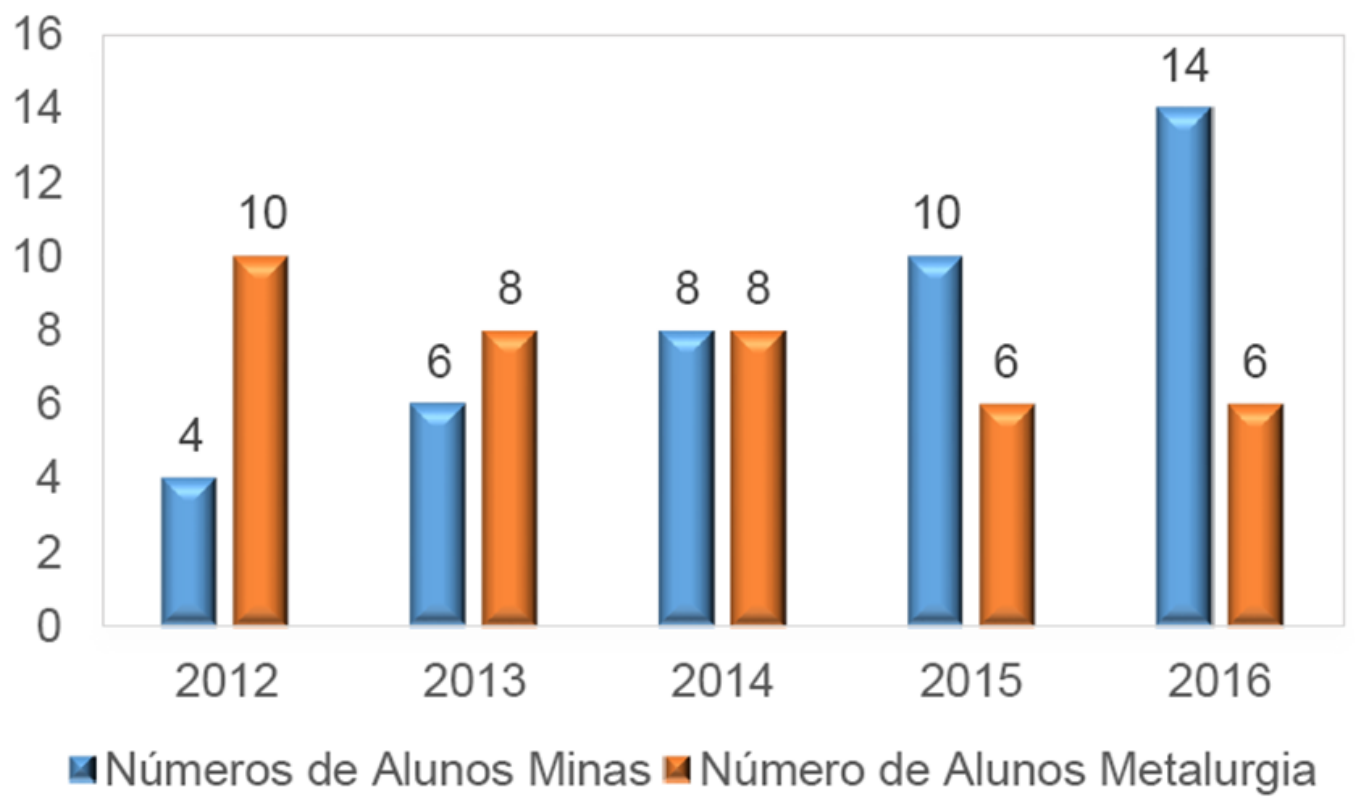

Figura 1. Número de alunos dos cursos de Engenharia de Minas e de Engenharia Metalúrgica participantes em atividades de iniciação científica.

A figura apresentada demonstra que a participação dos alunos em projetos de iniciação científica não é equivalente nos dois cursos estudados e, ao longo do tempo, não se constata um crescimento continuo nos dois cursos. Por exemplo, para o curso de Engenharia Metalúrgica foi observado um declínio no número de alunos bolsistas em relação ao ano de 2012. Somente o curso de Engenharia de Minas apresentou crescimento contínuo no número de projetos de pesquisa realizados. Essa variação pode estar ligada diretamente à política que a Instituição de Ensino adota em relação ao apoio pedagógico e financeiro no que diz respeito à pesquisa cientifica envolvendo alunos de graduação. Além disso, outro ponto relaciona-se com a constante troca e reposição anual do quadro de professores da UEMG, o que impacta diretamente na segurança e no desenvolvimento de projetos concebidos no segundo semestre letivo. Acredita-se que, com o aumento do quadro efetivo, o número de projetos possa aumentar, frente ao apoio e fortalecimento dos governos federais e estaduais. 
O número de publicações dos discentes de cada curso no período de 2012 até maio de 2016 é apresentado na Figura 2. Fica evidente que o número de publicações está bem baixo, se comparado ao número de bolsas concedidas a ambos os cursos. Percebe-se a necessidade dos professores incentivarem os alunos a publicarem artigos referentes aos projetos de pesquisa que desenvolvem. Também, pode-se inferir que a consolidação do corpo docente é um quesito importante para um maior incentivo aos discentes no aumento de suas publicações.

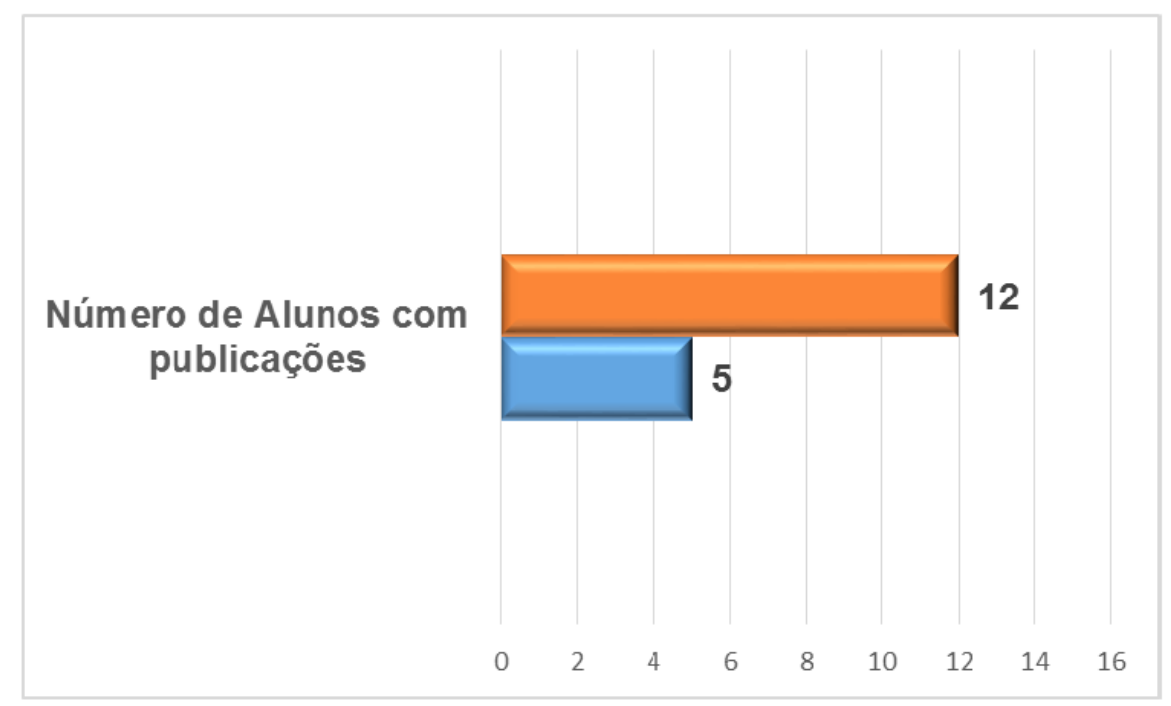

Figura 2. Publicações no período de 2012 a maio de 2016.

\section{CONCLUSÃo}

Os alunos dos cursos de engenharia da Universidade do Estado de Minas Gerais - Unidade João Monlevade - têm a oportunidade de realizar projetos de iniciação científica, a partir do momento que demonstram interesse e determinação em buscar o seu crescimento profissional. Ressalta se que a incorporação de um crescente número de professores efetivos pode resultar no aumento do número de projetos de pesquisa, uma vez que implica em uma maior qualificação e engajamento do corpo docente. Ao exercer tais atividades integradas ao ensino, o aluno tem a possibilidade de desenvolver suas habilidades e competências para o exercício da sua profissão, tornando-se mais qualificado para atender às exigências de um mercado cada dia mais competitivo.

Ao apresentar a realidade vivenciada pela universidade em relação à sua produção científica e ao analisar o número de programas de iniciação científica desenvolvidos pelos estudantes e professores dos cursos de engenharia da UEMG, verifica-se que existe uma disparidade entre os cursos e muitos desafios a serem encarados, como o desenvolvimento de propostas inovadoras, a participação mais efetiva dos alunos e o maior interesse na participação em projetos por parte dos professores, sejam eles designados ou efetivos. Adicionalmente, é sempre necessário o apoio da Instituição, focando em angariar mais recursos, não só financeiros relativos a bolsas, mas também em termos estruturais para a aquisição de equipamentos de laboratório necessários às referidas áreas de ensino de engenharia.

Diante disso, uma participação mais efetiva em todos os níveis da Instituição resultará, certamente, na maior eficiência e diversificação das atividades complementares nos seus cursos de engenharia e em uma formação profissional mais qualificada de seus engenheiros. 


\section{REFERÊNCIAS}

Bastos, F., Martins, F., Alves, M., Terra, M., Lemos, C. S. (2010). A importância da iniciação científica para os alunos de graduação em Biomedicina. Revista Eletrônica Novo Enfoque, Rio de Janeiro, v. 11, n. 11, pp. 61- 66.

Bazzo, A. W. (1998). Ciência, tecnologia e sociedade e o contexto da educação tecnológica. Florianópolis: Editora da USP. Documento disponível na internet: < www. campusoei.org/salactsi/bazzo02.htm> Acesso em 01/11/2016.

Conselho Nacional de Educação - CNE/CES. Resolução № 11 de 11 de março de 2002. Seção 1. Brasília, pp. 32.

Gomes, M. A. D. J., Gonçalves, M. D. F., Menin, P. A. H. (2004) A necessidade da iniciação científica para alunos de instituições de ensino superior particulares: a possibilidade de acesso crítico ao conhecimento como pretensão à excelência. In: Reunião Anual da associação nacional de pós-graduação e pesquisa em educação - ANPEd Caxambu. Petrópolis: Vozes, pp.222.

Simão, L. M., Guedes, M.C., Rodrigues, M.M.P. (1996). O papel da iniciação cientifica para a formação em pesquisa na pós-graduação. In: Simpósio de Pesquisa e Intercâmbio científico da associação nacional de pesquisa e pós-graduação e Psicologia - ANPPEP, Rio de Janeiro. Anais, pp. 111-113.

Tonini, A. M. (2007). Universidade Federal de Minas Gerais. "Ensino de Engenharia: as atividades acadêmicas complementares na formação do engenheiro". [Tese de Doutorado]. Faculdade de Educação, Belo Horizonte. 